GA-A20652

\title{
THE CHARGE EXCHANGE RECOMBINATION DIAGNOSTIC SYSTEM ON THE DIII-D TOKAMAK
}

by

P. GOHIL, K.H. BURRELL, R.J. GROEBNER, J. KIM, W.C. MARTIN, E.L. McKEE, and R.P. SERAYDARIAN

NOVEMBER 1991

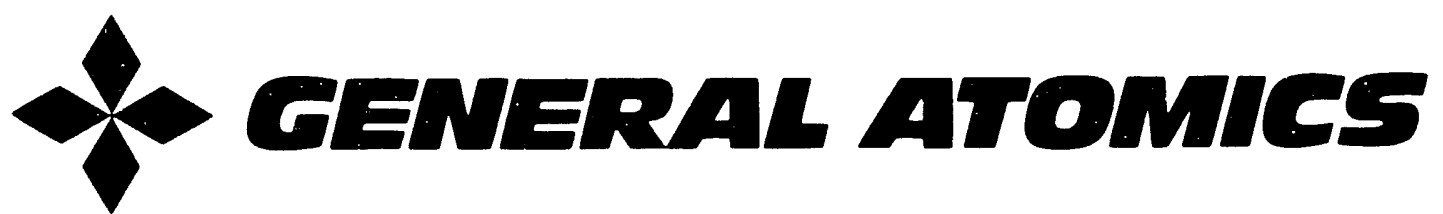




\section{DISCLAIMER}

This report was prepared as an account of work sponsored by an agency of the United States Government. Neither the United States Government nor any agency thereof, nor any of their employees, makes any warranty, express or implied, or assumes any legal liability or responsibility for the accuracy, completeness, or usefulness of any information, apparatus, product, or process disclosed, or represents that its use would not infringe privately owned rights. Reference herein to any specific commercial product, process, or service by trade name, trademark, manufacturer, or otherwise, does not necessarily constitute or imply its endorsement, recommendation, or favoring by the United States Government or any agency thereof. The views and opinions of authors expressed herein do not necessarily state or reflect those of the United States Government or any agency thereof. 


\title{
THE CHARGE EXCHANGE RECOMBINATION DIAGNOSTIC SYSTEM ON THE DIII-D TOKAMAK
}

by

P. GOHIL, K.H. BURRELL, R.J. GROEBNER, J. KIM, W.C. MARTIN, E.L. McKEE, and R.P. SERAYDARIAN

This is a preprint of a paper to be presented at the 14th Symposium on Fusion Engineering, September 30-October 3, 1991, San Diego, California, and to be printed in the Proceedings.

\author{
Work supported by \\ Department of Energy \\ Contract DE-AC03-89ER51114
}

GENERAL ATOMICS PROJECT 3466

NOVEMBER 1991

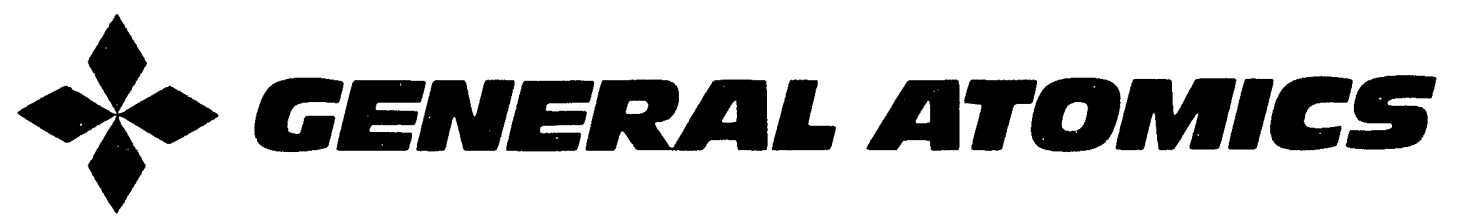




\title{
THE CHARGE EXCHANGE RECOMBINATION DIAGNOSTIC SYSTEM ON THE DIII-D TOKAMAK
}

\author{
P. Gohil, K.H. Burrell, R.J. Groebner, J. Kim \\ W.C. Martin, E.L. McKee, and R.P. Seraydarian \\ General Atomics \\ P.O. Box 85608 \\ San Diego, California 92186-9784
}

\begin{abstract}
The charge exchange recombination (CER) diagnostic system on the DIII-D tolamak is used to make spar tially and temporally resolved measurements of the ion temperature and toroidal and poloidal rotation velocities. This is performed through visible spectroscopic measurements of the Doppler broadened and Doppler shifted He II 468.6 nm, the C VI $529.1 \mathrm{~nm}$, and the B V $494.5 \mathrm{~nm}$ spectral lines which have been excited by charge exchange recombination interactions between the fully stripped ions and the neutral atoms from the heating beams. The plasma viewing optics comprises 32 viewing chords spanning a typical plasma minor radius of $63 \mathrm{~cm}$ across the midplane, of which 15 spatial chords span $4.2 \mathrm{~cm}$ at the plasma edge just within the separatrix and provide a chord-to-chord spatial resolution of $0.3 \mathrm{~cm}$. Fant camera readout electronics can provide a temporal resolution of $260 \mu$ per time slice, but the effective minimum integration time, at present, is $1 \mathrm{~ms}$ which is limited by the detected photon flux from the placma and the decay times of the phosphors used on the multichannel plate image intensifiers. Significant changes in the edge plasma radial electric field at the L-H transition have been observed, as determined from the CER measurements, and these results are being extensively compared to theories which consider the effects of sheared electric fieluts on plasma turbulence.
\end{abstract}

\section{Introduction}

Charge exchange recombination spectroecopy has been utilized at DIII-D to provide spatially and temporally resolved measurements of ion temperature and poloidal and toroidal ro tation velocities. Meanureconents of the ion temperature and toroidal rotation are sesential inputs to ion tranoport studies [1]. These measurements are also particularly relevant for determination of the radial electric field, $E_{p}$, which exhibits significant changes at the plasma edge at the transition from L-mode to $\mathrm{H}$-mode plasmas $\{2,3\}$ and which is important in theories of the $\mathrm{L}-\mathrm{H}$ transition $[4,5]$. Analyzing the behavior of the edge radial electric field at the $\mathrm{L}-\mathrm{H}$ transition is important in understanding the physics of the transition and H-mode plasmas. Therefore, lange efforts have been made at DIII-D to obtain high spatial and temporal resolution in these measurements together with greater accuracy and flexibility than previous systems. The capabilities of this diagnostic system have advanced from 16 spatial chords with a minimum spatial resolution of 3-4 cm per spatial point and 2 cas temporal resolution (6) to 32 chords with $0.7 \mathrm{~cm}$ minimum spatial resolution and $260 \mu s$ temporal resolution [7] to the present system comprising 32 chords with $0.3 \mathrm{~cm}$ minimum spatial resolution and $260 \mu$ semporal resolution. The greatest spatial resolution is achieved at the plasma edge across the mag. netic separatrix. This is essential to obtain detailed information on changes in $E_{r}$ which often occur within $2 \mathrm{~cm}$ from the separatrix at the time of the $\mathrm{L}-\mathrm{H}$ transition. Details on the CER system will be divided into sections describing: the layout of the viewing optics observing the plasma; the system for wavelength dispersion, light detection, and data acquisition: the spectroscopic observations and characteristics of the detected spectral emission: the data analysis and results from the system.

\section{Viewing Geometry and Optics}

The optical system viewing the plasma determines the spatial location and spatial resolution of the viewchords. The spatial localization of a particular chord is determined by the intersection of the viewing chord with a neutral heating beam. Figure I shovis a plan view of part of the DIII-D vessel indicating the chordal views through two pairs of neutral beams. The beamlines on DIII-D consist of pairs of neutral beams which intersect in the beamline drift duct at an angle of $8.7^{\circ}$ to each other. There are 32 chords: 17 chords view in a horizontal direction (i.e., in the toroidal plane), and 15 chords view in a vertical direction (i.e., in the poloidal plane).

The vertically viewing chords are not perfectly vertical because of limitations imposed by port location relative to the neutral beams. Therefore, a small component of the tangential plasma rotation is observed by the vertical views, which can be determined in the data anaiysis and removed so that a irue poloidal plasma rotatiou is obtained. In effect, the chords allow measurements clocer to $v_{\perp}$ than $v_{0}$ and this is helpful in the determination of $E_{p}$.

Seventeen of the viewing chords cover the range between 10 to $55 \mathrm{~cm}$ from the plasms center; seven of the seventeen are vertically oriented chords and ten are horizontal views. There are 15 edge chorda: 8 in the vertical direction and 7 in the horizontal direction [see Fig. 1(b)]. Furthermore, the verticaily and horizontally viewing chords are interleaved so as to form an alternating pattern of vertical and horizontal chords along the centerline of the heating beam. Therefore, the chord-to-chord separation is half that of the spatial separation between adjacent vertical (or adjacent horizontal) chords. The edge chords span a
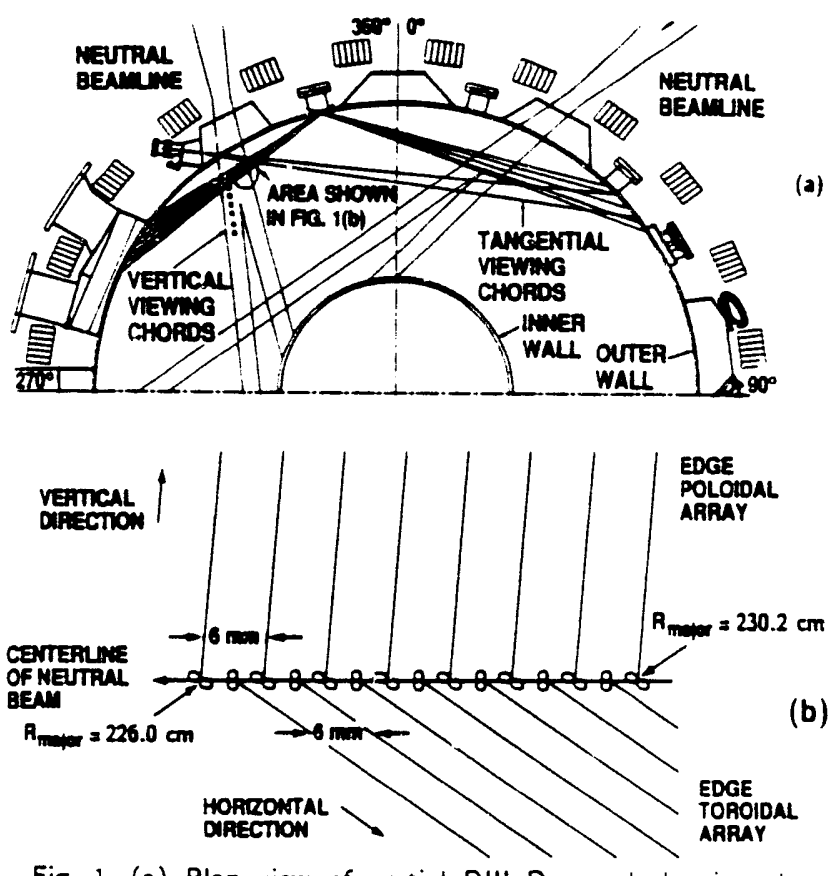

Fig. 1. (a) Plan view of partial DIII-D vessel showing the viewing chords through the neutral beams. The vertical viewing chords are indicated by the dots. (b) Edge views shown in more detail with the poloidal and tangential views interleaving along the centertine of the $330^{\circ}$ right source neutral beam. 


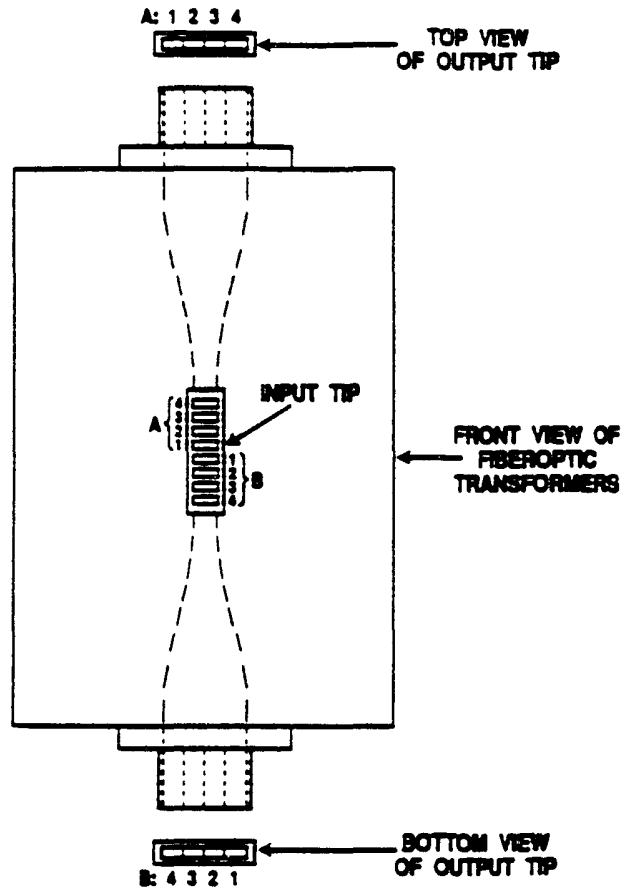

Fig. 2. Schematic of the coherent fiber optic coupling indicating the mapping of the spectrometer imace to the RETICON amroye.

total distance of $4.2 \mathrm{~cm}$, fired in space inside the vessel, but such that this spans the separatrix location at the midplane for most plasma configurations operated in DIII-D. The minimum chordto-chord separation is $0.3 \mathrm{~cm}$ with $0.6 \mathrm{~cm}$ being the separation between any pair of vertical or horizontal chords. These chords are aligned along the centerline of the $330^{\circ}$ right source neutral beam (left source and right source are references to the pair of beams that pass through each of the four beamlines on DIII-D). Since each pair of neutral beams is angled with respect to each other in the horizontal plane, the horizontally viewing chords can observe CER emission from both beams, whereas the vertical chords predominantly intersect one beam.

The viewing optics, which observe the CER emission, consist of a combination of mirrors and collection lenses. There are a total of 5 viewing systems (3 horizontal and 2 vertical) which cover the 32 chords. Most systems consist of a combination of a directing mirror or mirrors and a collection lens; in one case only a collect ion lens is required because of the direct viewing geometry available for observing a beam. The flat mirrors have aluminum $/ \mathrm{Mg}_{2}$ coatings on a fused silica substrate with maximum broadband reflectivity in the visible spectrum of interest. The fused silica lenses are plano-convex with anti-reflection coatings for the observed wavelengths for the edge chords. The optics are mounted such that the mirrors are located inside the vacuum vessel, but the lenses are positioned outside the vacuum window. This arrangement provides the best geometrical accessibility of the chords within the vacuum ressel.

The mirrors are well enclosed in protective shrouds, which have shutters, operated by linear actuators, over the view openings for the plasma. These shutters are automatically closed for helium glow discharge cleaning which is performed routinely between plasma discharges in DIII-D. Despite the shrouds and shutters, the aluminum $/ \mathrm{MgF}_{2}$ mirror coatings are constantly becoming cloudy after prolonged exposure to plasma operations. However, one of the mirrors presently being used has a BERAL coating and shows no obvious deterioration with time. Therefore, all in-vessel mirrors are now in the process of being replaced with substrates with BERAL coatings.
The light is focussed into silica core optical fibers which transmit the light into spectrometers which are placed outside the neutron shield wall for the DIII-D device. The distances between the lens and fibers for each system are set to provide the necessary demagnification with the correct $f$-number matching to the acceptance angle of the spectrometers. The typical demagnification is 6 . The optical fiber coupling mainly consists of bundles of dimension $2 \mathrm{~mm}$ by $1 \mathrm{~mm}$ comprising two $750 \mu \mathrm{m}$ core diameter optical fibers per bundle arranged vertically [toroidally for vertical views, see Fig. 1(b)]. Individual fibers of $1500 \mu \mathrm{m}$ core diameter are used for some of the chords viewing the central part of the planme. The bundles allowed for good light collection while maximizing the spatial resolution along the smaller dimension of the bundle. Within the chordal geometry, there are two pairs of chords observing the same major radii in essentially opposite directions, one pair in the vertical and the other in the horizontal, which are used as references to check the zero of rotation for the poloidal and toroidal rotation.

The spatial localization and resolution of the CER emistion is defined by the intersection of the viewing chords acrous the extent of the neutral beam crose-sectional profile. The spatial resolution is degraded by the curvature of the poloidal and toroidal flux surfaces within the region of intersection. The best spatial resolution is obtained when the viewing chord is tangent to the flux surface across the width of the neutral beam. Magnetic flux plots were examined for various single-null and double-null plasms configurations and the geometrical layout of the viewing chords was determined so as to produce the lowest uncertainty along the flux surface through the neutral beam croes sections. The full width half maximum dimensions of the neutral beam croes section are 14 and $30 \mathrm{~cm}$ in the horizontal and vertical directions, respectively. Also, the radius of curvature for the flux surfaces is smaller for the poloidal direction than for the toroidal direction. Therefore, the spatial resolution is worse for the poloidal chords than for the toroidal chords with the more central chords being worse than the edge chords because of the decreasing radius of curvature. Furthermore, the edge chords have the best tangency to the flux surfaces of all the chords. Typically, the central poloidal chords have a spatial resolution of $\pm 1.5 \mathrm{~cm}$, whereas the edge poloidal chords have a resolution of $\pm 0.5 \mathrm{~cm}$. The central toroidal chords have a resolution of $\pm 1 \mathrm{~cm}$, whereas the edge toroidal chord have a resolution of $\pm 0.3 \mathrm{~cm}$.

Calibration of the CER spatial locations inside the vessel is carried out in situ during vents for vessel entry. Large aluminum targets are used to represent the neutral beams. The targets contain the centerlines of each pair of neutral beams and essentially span the area between the inside and outside walls of the vessel. The targets are positioned according to reference

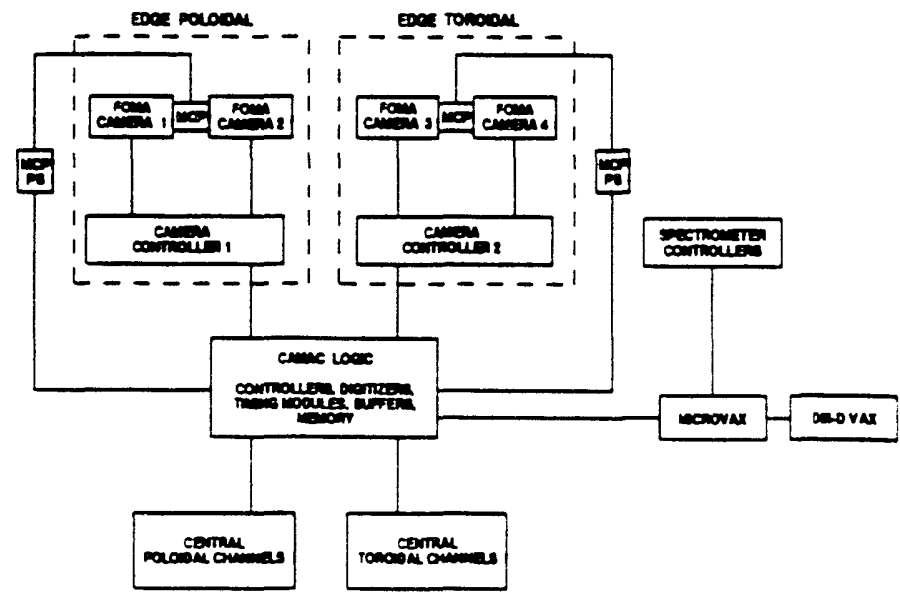

Fig. 3. Schematic of the electronic control hardware indicating the relationship of the main components. 
mariks which: (1) represent the crossover point of a pair of beams in the beamline drift duct, and (2) define the strike point of the right source beam on the inside wall as determined from the centroid of a heat flux pattern measured by calorimeters located on the inside wall. The optical fibers are illuminated in the reverse direction, i.e., from the spectrometers and into the vessel, and the viewing optics are adjusted to align the images of the fibers at the centerlines of the neutral beams at the desired major radii. This calibration is routinely performed during vents to verify that the chord locations have not changed during operations. In addition, a calibration is done whenever the mirrors are replaced. A more detailed spatial calibration is performed less frequently using cardboard targets which are placed at various locations inside the vessel and referenced to numerous parts of the vessel and the neutral beams. The chordal geometry determined in this manner provides accuracies to within a few mm for the major radii locations.

\section{Detection System}

The detection system encompasses the components which spectrally disperse, amplify, detect, and record the plasms emission. The light is transmitted through up to $22 \mathrm{~m}$ of optical fiber to an area located outside the neutron shield wall. The fibers are mountec onto the input slits of four spectrometers with eight chords per spectrometer. There are three $0.75 \mathrm{~m}$ f/6.8 and one $1 \mathrm{~m} f / 9$ Czenny-Turner spectrometers. Two of the $0.75 \mathrm{~m}$ spectrometers are used to disperse light from the edge chords with the central chords being split between the $0.75 \mathrm{~m}$ and the $1 \mathrm{~m}$ spectrometers. Each spectrometer has a 110 by $110 \mathrm{~mm}$ grating ruled with 1200 grooves per millimeter with the central spectrometers having gratings blased at $0.63 \mu \mathrm{m}$ and operated in first order for the observed spectral lines, whereas the edge spectrometers have gratings blazed at $1.2 \mu \mathrm{m}$ and are operated in second order for these lines. The central spectrometers have curved entrance slits with a 3 in radius of curvature

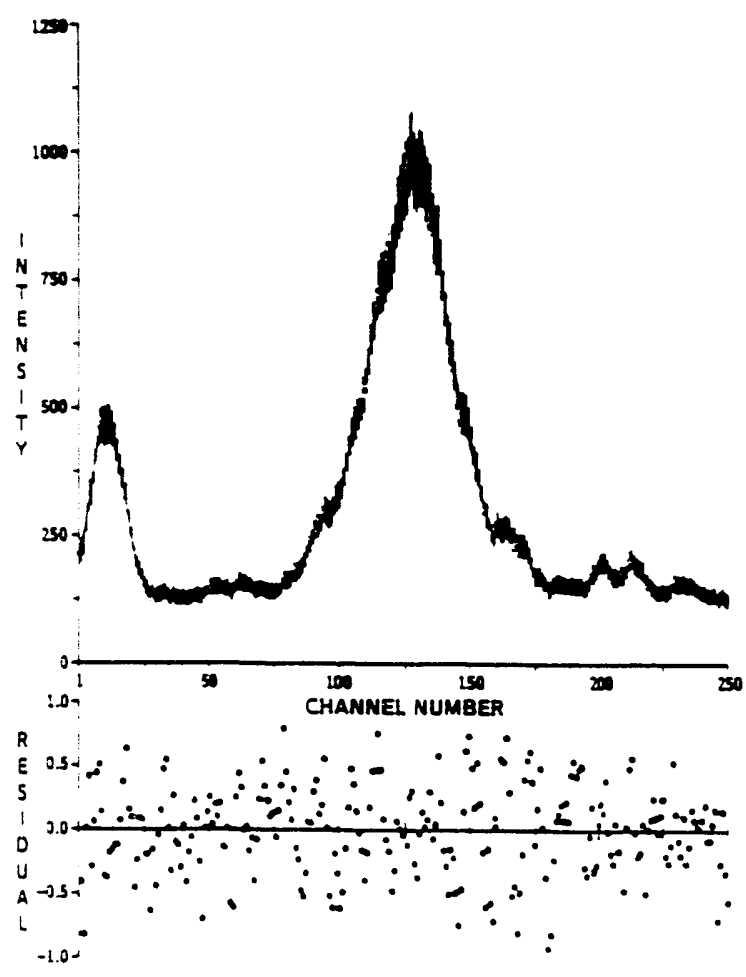

Fig. 4. Fitted spectrum of CVI emission in the presence of interfering lines. This is from an edge viewing chord observing in the toroidal direction. The lower part of the figure shows the departure of the fit from the experimental points in units of the standard deviation of the points.

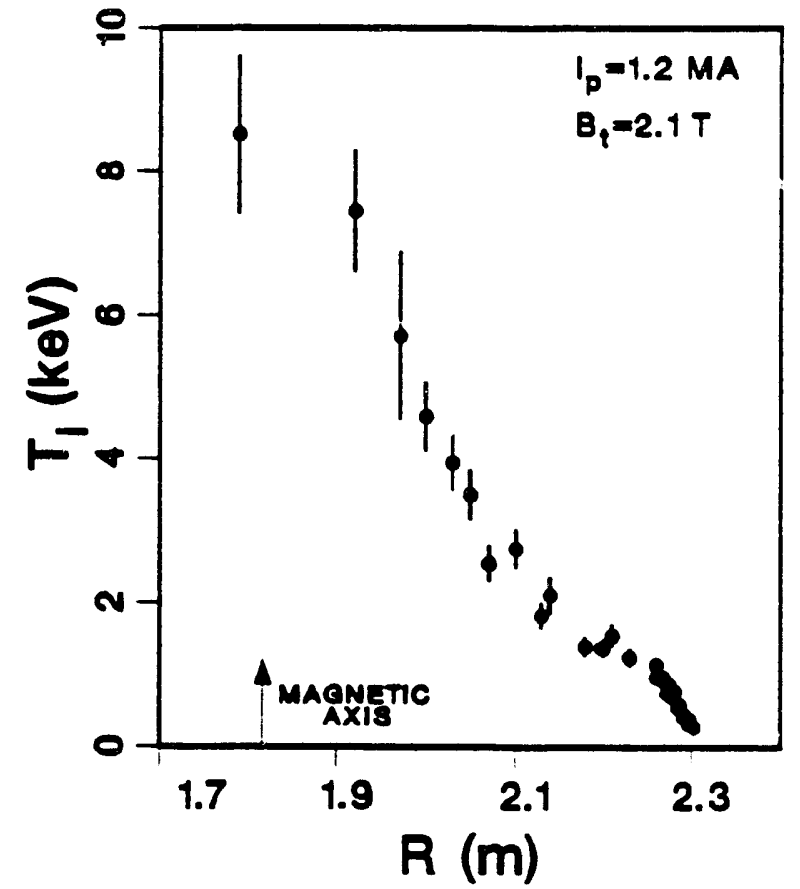

Fig. 5. The ion temperature profile as a function of the major radius for a VH-mode plasma discharge. The error bars in the $T_{i}$ of the edge chords are maller than the solid circles repreanting the data points.

and are normally operated at a width of $150 \mu \mathrm{m}$, whereas the edge spectrometers bave straight entrance slits and operated at $250 \mathrm{\mu m}$ slit widths. The optical fiber bundles are mounted in groups of eight vertically along the entrance slits at a separation of $3 \mathrm{~mm}$ center to center. The bundles have overall dimensions of $1 \mathrm{~mm}$ by $2 \mathrm{~mm}$ and are placed so that the individual fibers within the bundles are placed along the slit.

The exit plane of each spectrometer contains eight spectraily dispersed bands representing the eight spatial channels arranged one above the other. The spectral images are then passed through proximity focussed dual microchannel plate image intensifiers placed at the image plane of the spectrometers. The intensifiers are ITT $40 \mathrm{~mm}$ type F4150 tubes with an S20 photocathode and a tube resolution of typically 22 line pairs per millimeter. The tubes are coated with P20 phosphor.

Two types of phosphors were used initially. The central spectrometers used tubes with P20 phosphors and the edge spectrometers used tubes with $\mathrm{P} 46$ phosphor. The maximum tube gain was $10^{\circ}$ with the P20 phosphor and $2.7 \times 10^{5}$ with the P46 phosphors. The P20 phosphors have a temporal response which is initially fast $(<1 \mathrm{~ms})$ followed by a slow decay of the order of milliseconds. Furthermore, the temporal response can be further slowed down due to hysteresis effects from high levels of light intensity detected previously. The P46 phosphor was chosen because of its fast e-folding decay time of less than 1 us. However. in use and in subsequent extensive tests, the tubes with the P46 phosphors were found to be very nonlinear in their response. The linewidths of the wings of the line were very broad and varied with detected signal intensity. As the applied light intensity onto the tubes was increased, the relative amplitude of the widest Gaussian component of the spectral line (the spectrail line can be fitted by three Gaussians of different widths) increased as a function of the total number of counts detected at line center. This behavior occurred for a fixed tube gain and varying input light intensity or a fixed input light intensity with varying gain settings. The conclusion from numerous tests is that the tube was nonlinear as a result of the large current drain from the MCP needed to adequately drive th $=P 46$ phosphors, which have an efficiency ten times lower than P20. Consequently, the tubes 


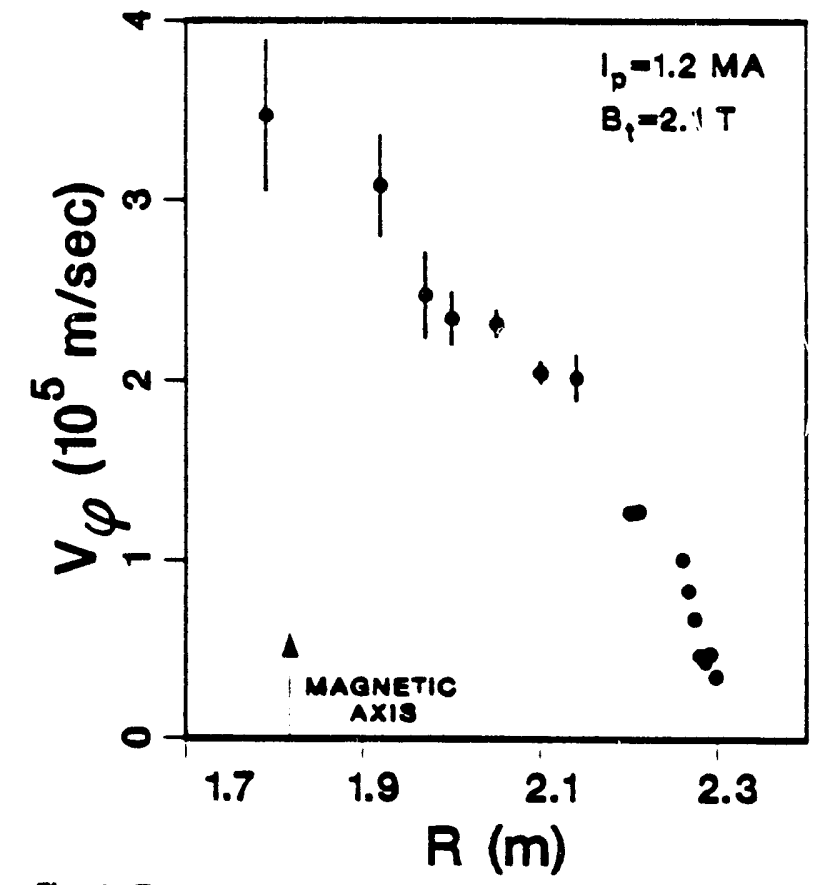

Fig. 6. The toroidal rotation velocity as a function of the major radius for the same discharge and time as Fig. 5.

with P46 phosphor were removed from the detection system and replaced with tubes with P20 phosphors.

The output from each intensifier tube is transmitted through a fiber optic image transformer to two 1024 pixel RETICON arrays. The image transformer consists of eight bands of tightly bundled $6 \mu \mathrm{m}$ core optical fibers with the bands being placed one above the other so as to be aligned with the eight spectrally dispersed bands of the spatial chords (see Fig. 2). The cross section of each coherent bundle is $1.60 \mathrm{~mm}$ by $6.35 \mathrm{~mm}$. As can be seen in Fig. 2, the eight bands of dispersed light are divided into two groups of four which are then arranged side by side so as to cover the length of the RETICON array. Two RETICON arrays are used and each band covers 256 pixels of the array with each pixel being $25 \mu \mathrm{m}$ wide.

The RETICON arrays are read by fast readout electronics in a system comprising camera heads, camera controllers, and commercially available CAMAC modules, which is referred to as a fast optical multichannel analyzer (FOMA) [8]. The control hardware is shown in Fig. 3. Each FOMA camera bead contains one RETICON 1024S linear photodiode array. The 512 odd photodiodes and the 512 even photodiodes are read by their own independent scanning circuit providing sequential odd and even outputs, where the amplitude is proportional to the light energy absorbed by the photodiode between consecutive scans. The minimum RETICON readout time is $500 \mathrm{~ns} /$ pixel for the edge system, which by using a two-phase clock and simultaneous readout of even and odd pixels gives a total readout time of $260 \mu \mathrm{s}$ per fhotodiode array. A flow of dry nitrogen gas through the camera head enclosure maintains a constant ambient temperature for the electrical components. Each camera controller contains the operating and timing logic, de power requirements and interlock needs for two camera beads and also provides output to waveform digitizers. The recharge and start signals from the controller to the camera heads are controlled by start of scan triggers generated by a Jorway 221 time sequence generator, which can produce up to $4 \mathrm{k}$ triggers in varying intervals with a resolution of $2 \mu \mathrm{s}$ within a $32 \mathrm{~s}$ long pulse train. The waveform digitizing hardware consists of TRAQ $4012 \mathrm{~A}$ analogto-digital system controllers, $2824 \quad 12$-bit $2 \mathrm{MHz}$ digitizers and $5004 \mathrm{~A} 1$ megaword memory modules.
The data acquisition and control software comprises FORTRAN programs and DCL commands on a DEC microVAX. An interactive program is used to control and modify hardware operational and timing parameters and to acquire, display, and transfer data to the DIII-D VAX data acquisitioning computer. This control extends to the high voltage power supplies for the microchannel plate image intensifiers which are gated and can be programmed for time-dependent gain voltage settings. The CAMAC timing and acquisitioning modules are initialized on reception of external asyachronous triggers from the central DIII-D timing system.

\section{Spectrosecopic Observations}

The Doppler broadened and Doppler shifted visible emisvion from the relevant ionic species (He, C, B) is used to determine the ion temperature, $T_{i}$, and poloidal, $v_{\theta}$, and toroidal, $v_{\phi}$, rotation velocities. The signal enhancement over normal plasma background results from CER interactions between the energetic deuterium atoms in the heating beams and fully stripped helium, carbon or boron ions, which are present as impurities in the plasma. The populations of the excited levels of the resultant hydrogen-like ionic states are mainly dependent on the cross section of the charge exchange process, the density of the fully stripped ions and the density of the neutral atoms in the region of interaction. The spectral lines of interest observed in the visible spectrum are $468.6 \mathrm{~nm}$ for He II $(n=4 \rightarrow 3), 529.1 \mathrm{~nm}$ for C VI $(n=8 \rightarrow 7)$ and $494.5 \mathrm{~nm}$ for BV $(n=7 \rightarrow 6)$. Helium is present in the plasma as a result of helium glow discharge cleaning which is routinely performed between plasma discharges in DIII-D. Boron is present in adequate concentrations for some time after boronization of the inside of the DIII-D vacuum vessel. Similarly, carbon is present as a result of the large area of carbon tiles covering the inside of the vessed, although the carbon concentration is significantly reduced after boronization. Typically, helium CER emission is analyzed for the chords viewing the central region of the plasma with boron and carbon emission being analyzed from chords observing the plama edge. Even though the fast readout capability of the system allows for a temporal resolution of $260 \mu \mathrm{s}$ per timeslice, the minimum useable integration time at present

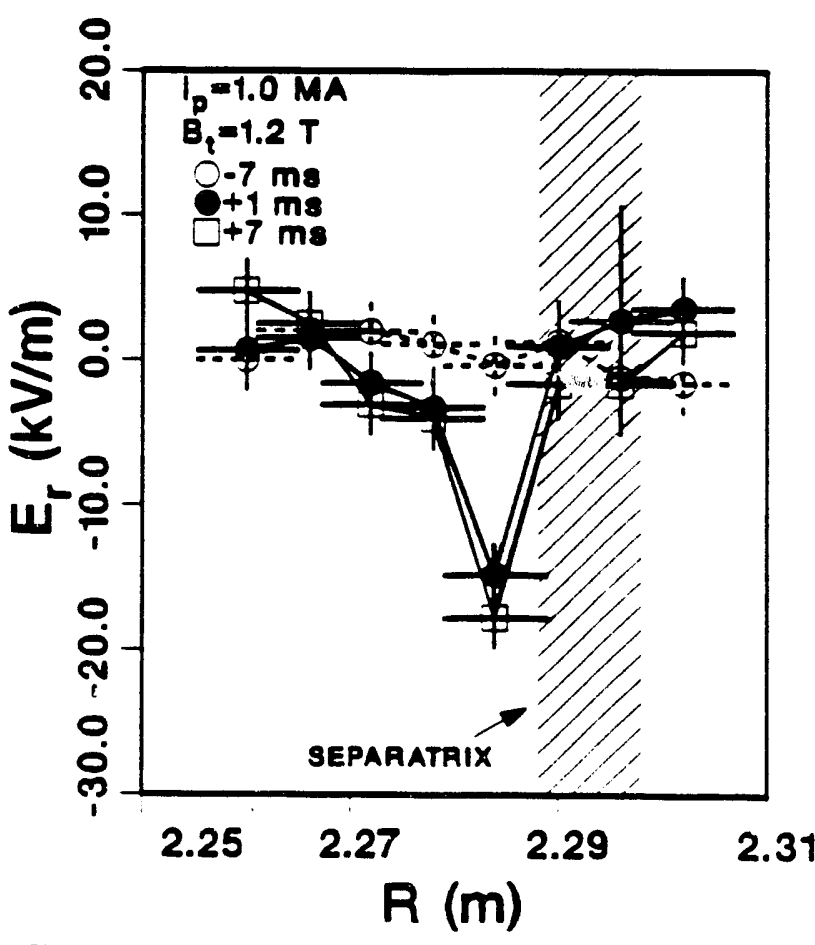

Fig. 7. The radial electric field. Er, at the plasma edge as a function of the major radius at specific times with respect to the $\mathrm{L}-\mathrm{H}$ transition. 
is $1 \mathrm{~ms}$; this is determined by the photon flux from the plasma. The observed spectral lines from these ions are sometimes complicated by the presence of polluting lines from the same species or lines from other impurity species such as oxygen. These interfering lines can make the data analysis laborious as the lines also have to be fitted as part of the spectral analysis. However, after boronization of the vessel, the intensity of oxygen lines was reduced to levels comparable to plasma background values and the CER spectral lines, especially for carbon, became relatively clean.

The ion rotation velocities are determined from the pixel shift of the peak of the fit to the CER line. The peak location of the CER line during the ohmic phase of the plasma discharge (i.e., no neutral beams) is used as the reference for the rotation measurements. In this respect, the zero of rotation is the obmic rotation and not an absolute measurement. However, corrections for any ohmic rotation can be made using observations from chords which view the same major radius, but in opposite directions both toroidally and poloidally. The addition of an absolute wavelength calibration of the system be tween every plasma discharge is presently underway. This would provide shot-to-shot wavelength references for each RETICON array through detection of known lines from a hollow cathode light source.

\section{Data Analysis and Results}

The determination of the Doppler width and Doppler shift of the spectral line for the seearurement of $T_{i}, v_{\theta}$, and $v_{\phi}$ utilizes nonlinear least-squares Gaussian fits to the peaks within the observed spectrum. The fits already include an instrumental response function, which is obtained from analysis of the $\mathrm{H}_{\mathrm{B}} \mathrm{I}$ $546.1 \mathrm{~nm}$ line produced by a low pressure mercury lamp. Any pressure broadening of the line is much smaller than the limiting instrumental widths. The instrumental width is best represented by the sum of three Gaussians. A white light calibration is also routinely performed to calibrate the intensity distribution across the detectors for each spatial channel. Furthermore, a relative chord-to-chord sensitivity calibration through the whole viewing optics and detection system is carried out by firing the neutral

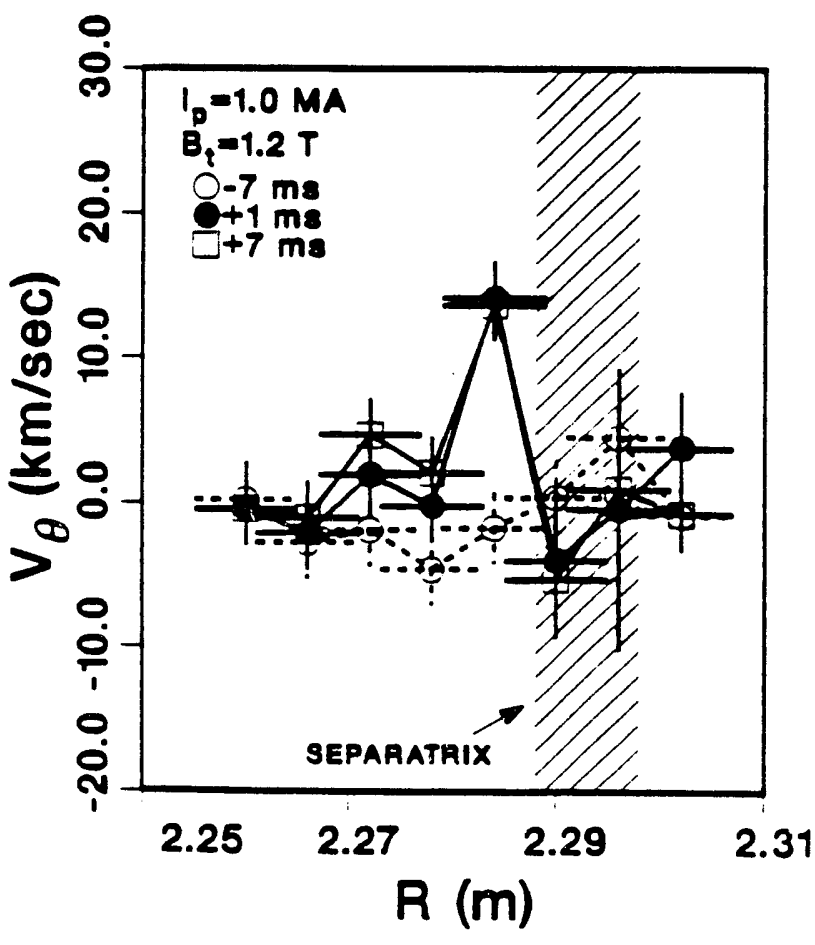

Fig. 8. The carbon poloidal rotation as a function of the major radius at the $L-H$ transition.

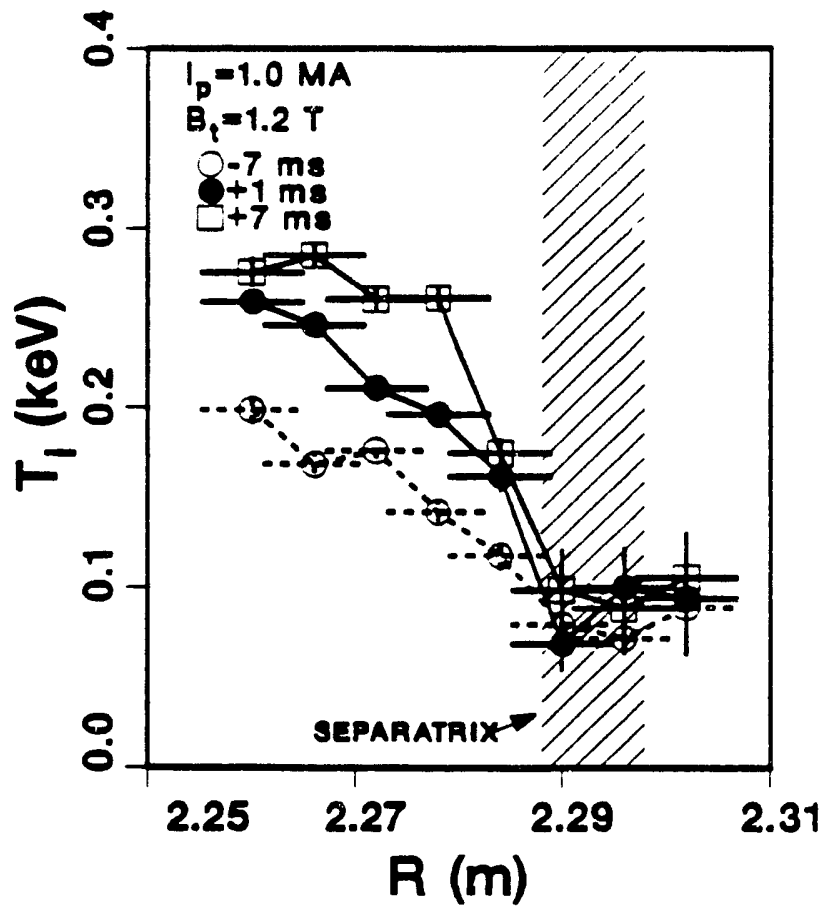

Fig. 9. The carbon temperature as a function of the major radius indicating the steepening of the edge $T_{i}$ gradient at the $L-H$ transition.

beams through a neutral gas at a given fill pressure in the DIII-D vessei.

The analysis code can simultaneousily fit up to 12 peaks together with a combination of predefined peaks for the common impurities found in DIII-D such as carbon, oxygen, and nickel. The complexity of the fit to the CER line is dependent on several factors. First, the number and characteristics of spectral lines from other impurity species and their proximity to the CER line effects the quality of the fit. Secondly, the fit can be complicated by spectral emission from the CER element resulting from collisional excitation which is typically emitted from the plasma edge at much lower temperatures. Furthermore, the fit is dependent on the spatial region of observation since the signal intensity is dependent on the density distribution of the observed ionic species across the plasma, the beam density as it penetrates through the plasma, and the plasma rotation profile across the plasma which effects the observed Doppler shift of the CER line and, hence, its relationship to other interfering lines. For example, Fig. 4 shows a case of a fitted C VI spectrum which has been complicated by the presence of several interfering lines. The fitting code can still determine the CER contribution since all the significant lines are included in the fit. In this case, the integrated area under the CER line is at least six times greater than the area under the next, most intense line. Analysis of the spectral data has become easier since the recent advent of boronization of the DIII-D vessel. After boronization, the spectra have cleaned up significantly, especially in the case of carbon. where one or two peak fits are now possible. Also, boron spectra are now also being analyzed.

The CER data is routinely used to produce ion temperature profiles for experimental analysis, especially for ion thermal transport analysis. Figure 5 shows an ion temperature profile as a function of the major radius. Only the points within the magnetic separatrix have been included. Note the high density of spatial points at the plasma edge. Also, the error bars on the edge points are smaller than the size of the point representing the chord. Figure 6 shows a toroidal rotation profile for the horizontal viewing chords for the same discharge as Fig. 5. The points span across the magnetic axis of the plasma. With the high edge spatial resolution, the edge $T$, gradients can now be accurately 
measured and. in the case of hot-ion H-mode plasmas, which essentially have high beam power input into low density target plasmas, edge $T_{1}$ gradients of the order of $i \mathrm{keV} \mathrm{cm}^{-1}$ have been measured. In addition, the study of the transition from $\mathrm{L}$-mode to $\mathrm{H}$-mode plasmas (referred to as L-H transition) has been one of the main driving forces in the upgrades to the CER system. Theoretical studies of the $\mathrm{L}-\mathrm{H}$ transition have focussed on the magnitude and/or shear of the radial electric field $[4,9,10]$ and the shear of the edge poloidal rotation :5! in suppressing microturbulence at the plasma edge, which would then lead to improved edige confinement. For example, neoclassical effects 11) need to be tested in comparison to the ion orbit loss model of Shaing, et al. 10!.

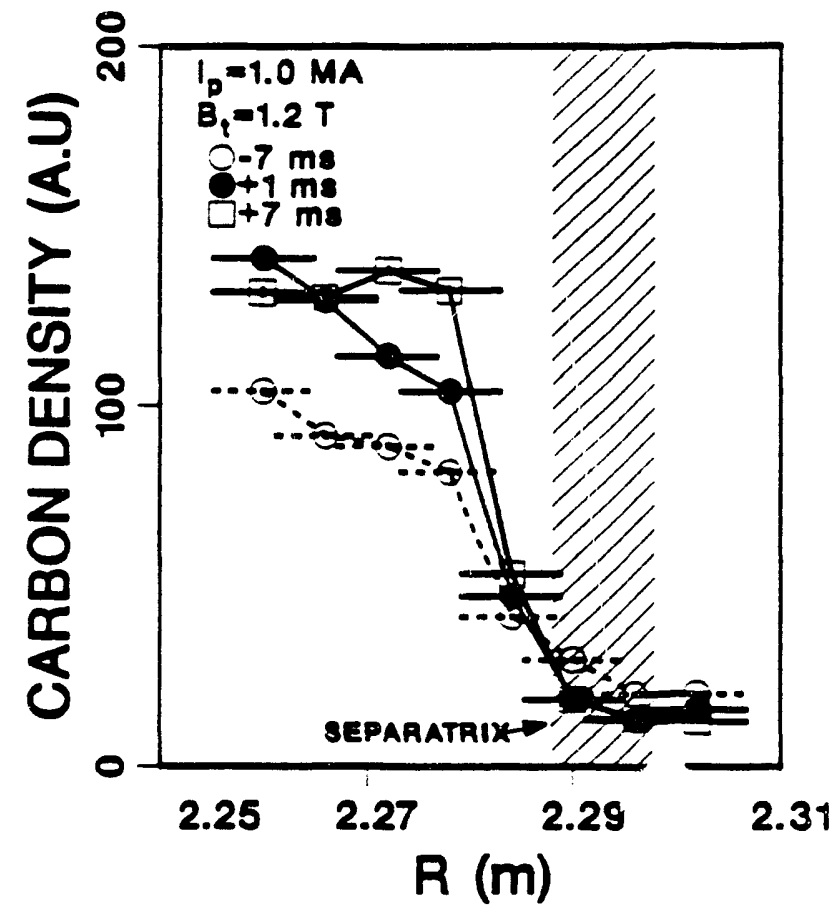

Fig. :2. The edge carbon density as a function of the major radius for the same plasm discharce and times as described in Fige. 7 through 9.

In the case of DIII-D, knowledge of the edge plasma ion pressure gradients and ion poloidal and toroidal rotation is used to determine the edge radial electric field from the lowest order force balance equation 12!. Figures 7 and 8 show the behavior of the edge electric field and the edge poloidal rotation, respectively, as a function of the major radius at specific times relative to the $\mathrm{L}-\mathrm{H}$ transition. The shaded region represents the uncertainty in the location of the separatrix as determined from .IHD equilibrium analysis. The key observations are that the snear in both the edge electric field, $E_{T}$, and the edge poloidal cotation. $v_{\theta}$, increase substantially within $2 \mathrm{cr}$ of the separatrix at the L-H transition. The structure of the $E_{+}$and $v_{\theta}$ profiles clearly show the need for the high spatial resolution at the plasma edge, which in :his case are represented by the spatia resolution of the edge vertical channels which have a spacing of
$0.6 \mathrm{~cm}$. The region of maximum shear in the edge electric field is also the region of suppression of the density fluctuation as indicated by reflectometry measurements at DIII-D 13!. This region is also indicated in Figs. 9 and 10 which show the edge carbon temperature and the carbon density as a function of the major radius at the same times. These figures indicate that the steepening of the profiles occurs over the region which corresponds to the shear in the radial electric field and marks the location of a transport barrier formed at the L-H transition. In this study, the overall conclusion is that the edge plasma microturbulence is suppressed by the shear in the edge radial electric field which forms at the L-B transition and this leads to the formation of an edge transport barrier, as indicated by steepening temperature and density profiles and improved edge confinement.

Further improvements to the CER system are being considered to address more physics issues, especially in the study of the $\mathrm{L}-\mathrm{H}$ transition. These include a single spatial channel operating at a fast time resolution of between 5-10 $\mu$ s to examine in greater temporal detail the changes that occur at the $\mathrm{L}-\mathrm{H}$ transition.

\section{Acknowiedgment}

This work was sponsored by the U.S. Department of Energy under Contract No. DE-AC03-89ER51114.

\section{Reference}

[1] Burrell, K.H., etah, in Plasma Physics and Controlled Nuclear Fusion Research (Proc. 13th Int. Conf. Washington, D.C., 1990) Vol. 1, LAEA, Vienna (1991) 123.

2) Groebner, R.J., et al, in Planma Physics and Controlled Nuclear Fusion Research (Proc. 13th Int. Conf. Washington, D.C., 1990) Vol. 1, LAEA, Vienna (1991) 453.

(3) Gohil, P., etal, "Study of Edge Electric Field and Edge Microturbulence at the L-B Transition in DIII-D, in Proc. 18th European Conf. on Controlled Fusion and Plasma Physics, Berlin, Germany, June 3-7, 1991; Vol. 1 (1991) 289

4) Shaing, K.C., and E.C. Crume, Jr., Phys. Rev. Lett. 63 (1989) 2369

5! Biglari, et al, Phys. Fluids B 2 (1990) 1.

6! Seraydarian, R.P., et al, Rev. Sci. lnst. 58 (1988) 1531.

7) Gohil, P., et al, Rev. Sci. Inst. 61 (1990) 2949.

8) Haskovec, J.S., et al., -FOMA - Fast Optical Multichannel Analyzer," GA Report GA-A19753, December 1989.

9! Itoh, S., and K. Itoh, Phys. Rev. Lett. 60 (1988) 2276.

10! Shaing, K., etad., Phys. Fluids B 2 (1990) 2162.

11) Kim, Y.B., etal., "Neoclassical Poloidal and Toroidai Retation in Tolamaks," Phys. Fluids B 3 (1991) 2050.

12! Einton, E.L., and R.D. Hazeltine, Rev. of Mod. Physics 48 (1976) 239.

13! Doyle, E.J., et al, Phys. Fluids B 3(8) (1991) 2300. 

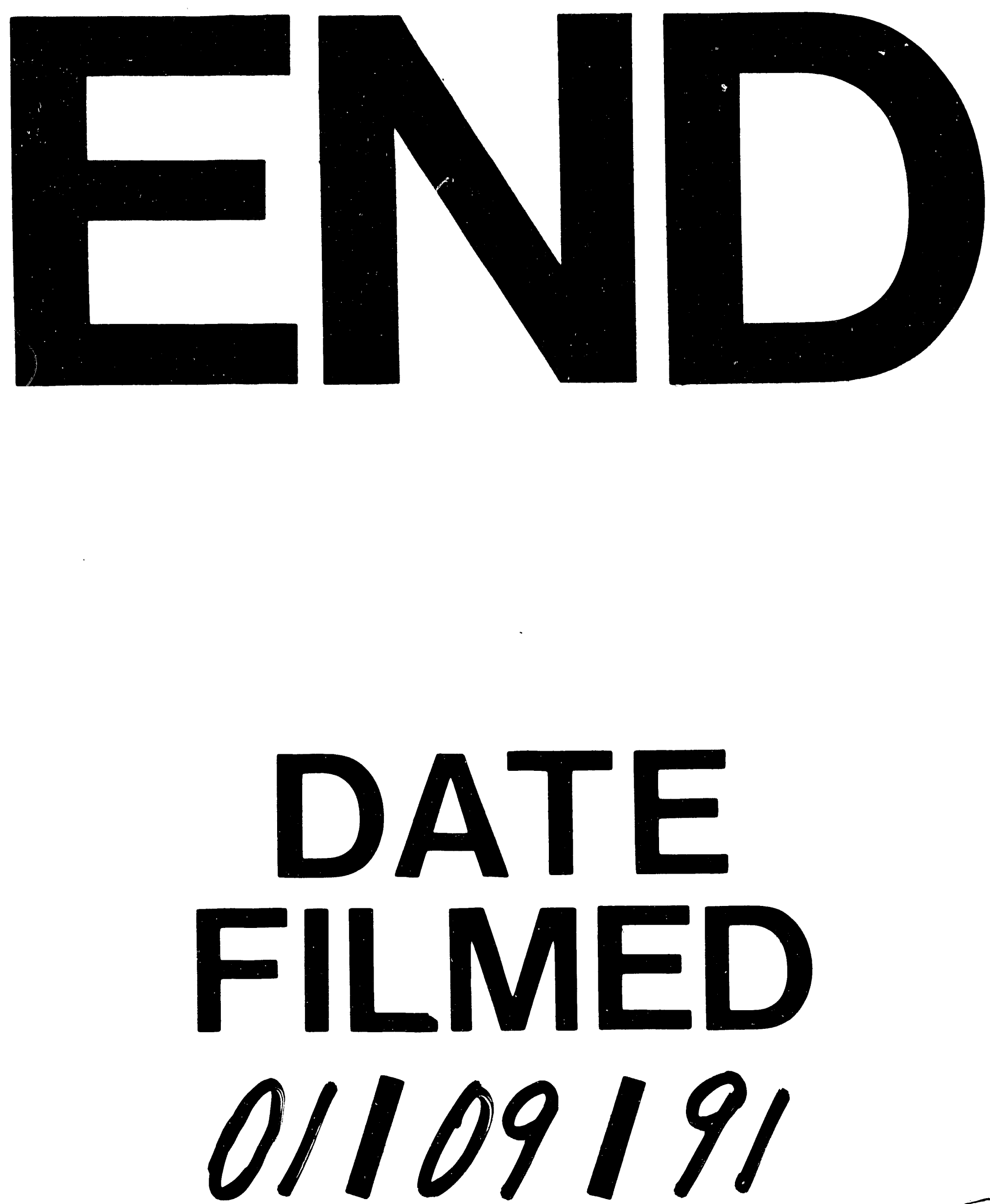

_ 
